\title{
Relation between risk of falls, sarcopenia and parameters assessing quality of skeletal muscles in a group of postmenopausal women
}

\author{
Maja Warzecha ${ }^{1}$, Jarosław Amarowicz ${ }^{1}$, Małgorzata Berwecka $^{1}$, Edward Czerwiński ${ }^{1}$, Anna Kumorek ${ }^{2}$ \\ ${ }^{1}$ Rehabilitation Clinic, Institute of Physiotherapy, Faculty of Health Sciences, Jagiellonian University Medical College, Krakow, Poland \\ ${ }^{2}$ Healthy Statistic, Krakow, Poland
}

\begin{abstract}
Introduction: Involutional changes that occur in skeletal muscle are a feature that characterizes the aging process. In women, age-related decreases in muscle mass and function of skeletal muscles occur more rapidly with the onset of menopause. Progressive muscle dysfunction has been directly linked with an increased probability of falls, fractures, disability and mortality.

Aim of the study: To assess the relationship between the risk of falls and parameters of skeletal muscle assessment in a group of postmenopausal women together with the identification of patients with sarcopenia.

Material and methods: This study was carried among 122 women over 60 years of age. Patients had their muscular system tested with the emphasis on the sarcopenia diagnosis using: Total Body Composition, handgrip and physical performance tests. Patients also underwent a questionnaire survey assessing occurrence of falls.

Results: The analysis showed an over 2-fold increase (OR $2.4 ; 95 \% \mathrm{Cl}, 1.02-5.56)$ in risk of falls in a year among subjects with decreased muscle mass. No such correlation was noted with parameters such as falls in the last 12 months and decrease of muscle strength as well as physical performance. Sarcopenia is more likely to be diagnosed with European Working Group on Sarcopenia in Older People (EWGSOP1) criteria than EWGSOP2 (updated in 2018$)(18 \%$ vs. $4.1 \%$ respectively). The increased risk of falls has not been proven in women with sarcopenia.
\end{abstract}

Conclusions: The decrease of muscle mass is significantly correlated with the risk of falls in the last year in postmenopausal women. Impact of sarcopenia on the risk of falls depends on diagnostic criteria.

Key words: handgrip, muscle mass, physical performance, TUG, falls.

\section{Introduction}

Involutional changes that occur in the composition of a human body are a feature that characterizes the aging process. They include, among others, skeletal muscle where the loss of muscle mass begins in the third decade of a person's life span, reaching significant losses after 50 years of age [1] with an average loss of $1.59 \%$ in men and $2.02 \%$ in women for a period of 4 years [2]. It is estimated that for the age group of 40-80 years the loss of muscle mass reaches $30-60 \%$ [3]. Muscle strength reaches its peak in the early adult life, and is then followed by a period of maintenance prior to a constant decline observed as aging progresses [4]. Average annual decline in muscle strength for a person over 65 years of age is 0.789 and $1.239 \mathrm{~kg}$ for men and women respectively (measured with handgrip) [2]. Studies show that physical performance in a group of healthy female subjects within the age of 68-82 years decreases by an average of $11 \%$ per 3 years (measured with gait speed) [5]. The same studies report a decline in walking speed, i.e. $0.019 \mathrm{~m} / \mathrm{s}$ for men and $0.025 \mathrm{~m} / \mathrm{s}$ for women [2]. All involutional changes that affect skeletal muscles (appendicular lean muscle mass/height ${ }^{2}$ - ASMM, handgrip, gait speed) occur more rapidly in women [2]. This is caused by a number of non-sex-related factors, i.e. lower regeneration ability resulting in aging of the satellite cells, decreased myocyte regeneration and synthesis of the muscle protein. Involutional changes are also caused by sex-related factors such as the age-associated decrease in sex hormones. With the onset of menopause the decrease in mass and strength of skeletal muscles is more rapid [6]; therefore there is a need for additional care of postmenopausal women in relation to their risk of sarcopenia.

It is estimated that over the age of 60 years sarcopenia affects up to $10 \%$ of the world's population [7]. It has been shown that sarcopenia covers $1-29 \%$ of respondents as well as $14-33 \%$ of patients within the healthcare institutions, depending on the definition 
used [8]. In 2010 the European Working Group on Sarcopenia in Older People (EWGSOP1) published a definition of sarcopenia along with a diagnostic algorithm, updated in 2018 (EWGSOP2), following an increasingly growing amount of new evidence in the field. According to the new criteria of EWGSOP2 sarcopenia is defined as an age-related disease characterized by a loss of muscle mass and impaired function (strength and physical performance). This progressive disorder is accompanied by an increased risk of falls, fractures, disability and death $[9,10]$.

Among elderly falls are the major cause of trauma and the second most common cause of trauma-related death. It is estimated that 1 in 3 people over 65 years of age and 1 in 2 over 85 years will experience a fall within a year $-10 \%$ of which will result in hospitalization [11]. According to the data from the National Institute of Public Health, 4451 cases of fall-related deaths were reported in 2016, which constitutes a 19\% increase in the death rate since 2010 [12]. Women are significantly more likely to experience falls and hospitalizations following them [11], which, together with the advanced pace of involution changes in the muscular system of postmenopausal women, has become the premise to undertake more detailed studies on the topic. The authors believe that this study will provide medical staff with guidance on the necessity of early implementation of fall prevention in women at risk of sarcopenia, which might have a direct impact on reducing the incidence of injuries and hospitalization due to falls as well as developing a new model for dealing with women at risk.

\section{Aim of the study}

The aim of the study was to assess a relationship between the risk of falls and parameters of skeletal muscle assessment in a group of postmenopausal women together with the identification of patients with suspected sarcopenia (presarcopenia) and sarcopenia using EWGSOP diagnostic criteria from 2010 and 2018.

\section{Material and methods}

This cross-sectional study was carried in accordance with the principles of the Helsinki Declaration of the World Medical Association after obtaining consent from the Bioethics Committee among patients of the Krakow
Medical Centre (KCM), who gave their informed consent to participate in the study. 122 women over 60 years of age were qualified to the study group where all assessments required by the study protocol were done. The study group was characterized by an average age of 69 years (60-81; SD 5.4), height $152 \mathrm{~cm}$ (140.5-172.2; SD 6.2), weight $70.1 \mathrm{~kg}(38.2-115,6$; SD 14.7) and body mass index (BMI) $28 \mathrm{~kg} / \mathrm{m}^{2}$ (15.4-44.0; SD 5.5). All the tests were conducted within one visit during outpatient hours from 8 a.m. to 4 p.m. Due to the hourly span, patients' tests were conducted without guidelines regarding meal times or the necessity of defecation. Patients had their muscular system tested with the emphasis on the sarcopenia diagnosis using: Total Body Composition (Hologic Horizon Bone Densitometer, Horizon W, Bedford, USA), measurement of muscle strength - handgrip (Baseline hydraulic dynamometer, 12-0240, NY, USA) and physical performance tests (4 meter gait speed test and Timed Up and Go - TUG). Immediately before the densitometric examination each patient underwent a measurement of body height and weight at a calibrated stadiometer and certified scales and was instructed to remove metal parts to avoid unreliable results. The two measurements of handgrip were performed in a standing position with shoulders extended along the torso, elbow joints bent at an angle of 90 degrees and forearm in a neutral position. The higher handgrip result (out of two try-outs) achieved by the study subjects was taken into analysis. For the TUG test individuals were asked to rise from a standard chair, walk to a marker located 3 meters away, turn around, walk back and sit down again. To measure the gait speed, a 6-meter section was determined although the time measurement was executed only for a central 4-meter distance. The time was measured in both gait tests using an electronic stopwatch.

Two sets of cut-off points were used based on the EWGSOP definition from 2010 (for women: ASMM/height ${ }^{2}<5.5 \mathrm{~kg} / \mathrm{m}^{2}$, handgrip $<20 \mathrm{~kg}$, speed $<0.8 \mathrm{~m} / \mathrm{s}$, TUG > $10 \mathrm{~s}$ ) and 2018 (for women: ASMM/ height $^{2}<5.5 \mathrm{~kg} / \mathrm{m}^{2}$, handgrip $<16 \mathrm{~kg}$, speed $<0.8 \mathrm{~m} / \mathrm{s}$, TUG > $20 \mathrm{~s})$ for comparison. EWGSOP1 criteria allow one to identify presarcopenia in a situation when only the muscle mass is decreased and sarcopenia when ASMM loss is accompanied by a reduction in handgrip and/or physical performance. Updated guidelines of EWGSOP2 recognize suspected sarcopenia on the basis

Table 1. Diagnostic criteria for sarcopenia defined by EWGSOP1 [9] and EWGSOP2 [10]

\begin{tabular}{|c|c|c|}
\hline \multirow[t]{2}{*}{ Diagnosis } & \multicolumn{2}{|c|}{ Diagnostic criteria for sarcopenia } \\
\hline & EWGSOP1 & EWGSOP2 \\
\hline Presarcopenia/Suspected sarcopenia & $\downarrow$ ASMM & $\downarrow$ handgrip \\
\hline Sarcopenia & $\downarrow$ ASMM + handgrip/gait speed (TUG/4 m) & $\downarrow$ handgrip + ASMM \\
\hline Severe sarcopenia & $\downarrow$ ASMM + handgrip + gait speed (TUG/4 m) & $\downarrow$ handgrip + ASMM + gait speed (TUG/4 m) \\
\hline
\end{tabular}


Table 2. Detailed group characteristics with the emphasis on the history of falls in the last 5 years

\begin{tabular}{lccc}
\hline Variables & $\begin{array}{c}\text { Fallers } \\
(n=60)\end{array}$ & $\begin{array}{c}\text { Non-fallers } \\
(n=55)\end{array}$ & $p$ \\
\hline Age (years) & 69.8 & 68.4 & 0.21 \\
\hline Height $(\mathrm{cm})$ & 157.6 & 158.9 & 0.27 \\
\hline Weight $(\mathrm{kg})$ & 71.2 & 68.1 & 0.31 \\
\hline BMI $\left(\mathrm{kg} / \mathrm{m}^{2}\right)$ & 28.7 & 26.9 & 0.12 \\
\hline ASMM $\left(\mathrm{kg} / \mathrm{m}^{2}\right)$ & 6.1 & 5.8 & 0.41 \\
\hline Handgrip $(\mathrm{kg})$ & 21.7 & 23.0 & 0.19 \\
\hline TUG $(\mathrm{s})$ & 11.7 & 10.5 & 0.91 \\
\hline 4 m gait speed $(\mathrm{s})$ & 3.7 & 3.4 & 0.86 \\
\hline Number of falls $(n)$ & 122 & - & - \\
\hline ASMM
\end{tabular}

ASMM - appendicular lean muscle mass/height ${ }^{2}$, TUG - Timed Up and Go test

of a reduced handgrip and confirmed diagnosis of sarcopenia when accompanied by the loss of muscle mass measured with ASMM index (Table 1).

In addition, patients underwent a questionnaire survey assessing occurrence of falls in the last 12 months and 5 years. According to the WHO definition a fall is defined as a sudden incident, an unexpected loss of balance while walking or performing other activities, which results in the patient finding himself on the ground. Due to this definition falls analyzed in this study included only those that happened from the height of a standing position. Seven patients were excluded from the analysis due to incorrect or ineligible completion of the questionnaire on falls. Statistical analysis was carried out with Statistica 13 using tests, i.e. $\chi^{2}$ (Pearson), Student's $t$, Mann-Whitney $U$, comparison of average values and analysis of variance (ANOVA). Values of $p<0.05$ were considered to be statistically significant.

\section{Results}

Women in the study group were characterized by average values of parameters assessing skeletal muscles at the level of: $6.0 \mathrm{~kg} / \mathrm{m}^{2}$ for ASMM (4.3-9.5; SD 0.9), $22.2 \mathrm{~kg}$ for handgrip (3-38; SD 6.1), 11.5 seconds for the TUG test (5-45.5; SD 7.0) and 3.7 seconds for gait speed assessment (1.5-14.1; SD 1.6). Sixty women (49.2\%) suffered at least one fall in the last 5 years with a to-

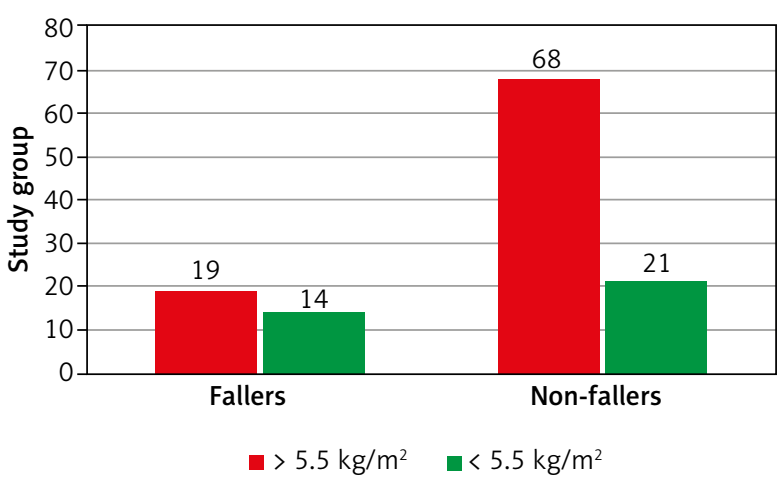

Fig. 1. Ratio of women with a fall in the last year to those without a fall in relation to muscle mass

tal of 122 falls for the whole study group. Both in the fallers and non-fallers the most commonly diagnosed co-morbidities were rheumatic ( $85 \%$ vs. $81 \%)$, cardiological (35\% vs. $46 \%$ ) and endocrine (35\% vs. $22 \%$ ) diseases. Due to the prevalence of comorbidities, the respondents who fell and those who did not most often took medications for the cardiovascular system $(25 \%$ vs. $36 \%$ ), for the thyroid ( $12 \%$ vs. $11 \%$ ) and steroids ( $8 \%$ vs. $15 \%)$. The study did not make a detailed assessment of the impact of comorbidities on the risk of falls. A detailed description of the studied subjects divided into the falling group and non-falling group is presented in table 2. No significant differences were observed between the groups regarding age, anthropometric indexes (weight, height, BMI) or parameters assessing the quality of skeletal muscles (Table 2).

The analysis showed that the risk of falls among the studied subjects was statistically significant in relation to their decreased muscle mass (i.e. ASMM $<5.5 \mathrm{~kg} / \mathrm{m}^{2}$ ). The odds ratio in the studied group showed over a 2-fold increase (OR 2.4; 95\% Cl, 1.02-5.56) in subjects with a decreased muscle mass when compared to those with an ASMM healthy value defined by the EWGSOP guideline (Table 3). The ratio between non-fallers and those who have sustained a fall in the last year was $1.5: 1$ in those with a decreased muscle mass (Fig. 1).

There was no correlation between falls in the last 12 months and decrease of muscle strength as well as physical performance assessed with the TUG test and $4 \mathrm{~m}$ gait speed test. Results were similar regardless the

Table 3. Relation between the risk of falls in the last year and last 5 years and parameters assessing quality of skeletal muscles in accordance with EWGSOP1 and EWGSOP2 criteria

\begin{tabular}{lcccc}
\hline $\begin{array}{l}\text { Muscular system } \\
\text { parameters }\end{array}$ & \multicolumn{2}{c}{ Falls 1 year (OR [95\% CI]) } & \multicolumn{2}{c}{ Falls 5 years (OR [95\% CI]) } \\
\cline { 2 - 5 } & EWGSOP1 & EWGSOP2 & EWGSOP1 & EWGSOP2 \\
\hline$\downarrow$ Muscle mass & $2.4(1.02-5.56)^{*}$ & $2.4(1.02-5.56)^{*}$ & $1.07(0.48-2.38)$ & $1.07(0.48-2.38)$ \\
\hline$\downarrow$ Handgrip & $1.00(0.41-2.40)$ & $0.41(0.08-1.95)$ & $1.57(0.70-3.55)$ & $2.00(0.56-7.05)$ \\
\hline$\downarrow$ Performance (TUG) & $0.53(0.21-1.31)$ & $0.36(0.04-3.06)$ & $1.31(0.59-2.88)$ & $1.82(0.33-10.76)$ \\
\hline$\downarrow$ Performance (4 m) & $0.22(0.02-1.78)$ & $0.22(0.02-1.78)$ & $4.15(0.84-20.48)$ & $4.15(0.84-20.48)$ \\
\hline
\end{tabular}

EWGSOP - European Working Group on Sarcopenia in Older People, TUG - Timed Up and Go test, ${ }^{\star} p<0.05$ (after Sheskin) 
Table 4. Prevalence of sarcopenia in the study group in accordance to the EWGSOP1 and EWGSOP2 criteria

\begin{tabular}{lccccc}
\hline Diagnosis & \multicolumn{2}{c}{ EWGSOP1 } & \multicolumn{2}{c}{ EWGSOP2 } & \multirow{2}{*}{$p$} \\
\cline { 2 - 5 } & $n$ & $\%$ & $n$ & $\%$ & 0.255 \\
\hline Presarcopenia/Suspected sarcopenia & 16 & 13.1 & 8 & 6.6 & $<0.001$ \\
\hline Sarcopenia & 22 & 18 & 5 & 4.1 & - \\
\hline Norm & 84 & 68.9 & 109 & 89 & 89 \\
\hline
\end{tabular}

EWGSOP - European Working Group on Sarcopenia in Older People

Table 5. Characteristics of the study group with the emphasis on sarcopenia defined using the EWGSOP1 and EWGSOP2 criteria

\begin{tabular}{|c|c|c|c|c|c|c|}
\hline \multirow[t]{2}{*}{ Variables (average) } & \multicolumn{2}{|c|}{ EWGSOP1 } & \multirow{2}{*}{$\begin{array}{c}\text { Norm } \\
(n=84)\end{array}$} & \multicolumn{2}{|c|}{ EWGSOP2 } & \multirow{2}{*}{$\begin{array}{c}\text { Norm } \\
(n=109)\end{array}$} \\
\hline & $\begin{array}{l}\text { Presarcopenia } \\
\quad(n=16)\end{array}$ & $\begin{array}{l}\text { Sarcopenia* } \\
\quad(n=22)\end{array}$ & & $\begin{array}{l}\text { Suspected } \\
\text { sarcopenia } \\
\quad(n=8)\end{array}$ & $\begin{array}{c}\text { Sarcopenia* }^{*} \\
(n=5)\end{array}$ & \\
\hline Age (years) & 67.8 & 70.6 & 69 & 70.6 & 68.8 & 69.1 \\
\hline BMI $\left(\mathrm{kg} / \mathrm{m}^{2}\right)$ & 23.0 & 22.8 & 30.3 & 29.9 & 23.3 & 28.1 \\
\hline $\operatorname{ASMM}\left(\mathrm{kg} / \mathrm{m}^{2}\right)$ & 5.1 & 5.0 & 6.4 & 6.1 & 4.6 & 6.04 \\
\hline Handgrip (kg) & 23.2 & 19.7 & 22.6 & 10.3 & 14.2 & 23.4 \\
\hline TUG (s) & 8.0 & 13.9 & 11.6 & 19.8 & 15.8 & 10.7 \\
\hline 4 m gait speed (s) & 3.0 & 3.7 & 3.8 & 4.7 & 4.5 & 3.6 \\
\hline $\begin{array}{l}\text { Number of fallers in the } \\
\text { last } 12 \text { months }(n[\%])\end{array}$ & $5(31.3)$ & $8(36.4)$ & $19(22.6)$ & 0 & $2(33.3)$ & $31(28.4)$ \\
\hline $\begin{array}{l}\text { Number of fallers in the } \\
\text { last } 5 \text { years }(n[\%])\end{array}$ & $8(50)$ & $11(50)$ & $41(48.8)$ & $4(50)$ & $4(80)$ & $52(47.7)$ \\
\hline
\end{tabular}

Table 6. Risk of falls in the last 12 months as well as in the last 5 years in relation to the prevalence of sarcopenia

\begin{tabular}{lcc}
\hline Falls & \multicolumn{2}{c}{ Sarcopenia [OR $(95 \% \mathrm{Cl})]$} \\
\cline { 2 - 3 } & EWGSOP1 & EWGSOP2 \\
\hline 12 months & $1.71(0.64-4.5)$ & $1.84(0.29-11.59)$ \\
\hline 5 years & $1.03(0.41-2.6)$ & $3.92(0.42-3.56)$ \\
\hline
\end{tabular}

EWGSOP - European Working Group on Sarcopenia in Older People

cut-off points used (EWGSOP1 and EWGSOP2). Risk of falls was also not significantly increased for falls in the last 5 years in those with decreased skeletal muscle quality parameters (Table 3 ).

Presarcopenia was diagnosed in $13.1 \%$ (using EWGSOP1) and suspected sarcopenia was observed in $6.6 \%$ (EWGSOP2) of the study group. Sarcopenia (including severe sarcopenia) was found in $18 \%$ and $4.1 \%$ of patients respectively. Following these results it was proven that sarcopenia is 4 times more likely to be diagnosed with EWGSOP1 when compared to the updated criteria from 2018 (Table 4).

Detailed characteristics of the study group, specifying patients with a suspected sarcopenia, presarcopenia and sarcopenia assessed with the EWGSOP criteria from 2010 and 2018, are presented in Table 5. Study results show that women with sarcopenia were more likely to sustain a fall in the last 12 months regardless of the EWGSOP criteria that were used to define it (36.4\% and $33.3 \%$ respectively) when compared to healthy subjects. Similar results were observed to those with sarcopenia in relation to falls in the last 5 years $(50 \%$ and $80 \%$ respectively) (Table 5). Since the parameters of the quality of the skeletal muscles are components of the definition of sarcopenia, they were not assessed separately for statistical significance.

Further analysis involved assessment of risk of falling in a group of patients with sarcopenia. An increased risk of falls in the last 12 months was observed in subjects with sarcopenia regardless of the EWGSOP (2010 and 2018) criteria that were used as a definition (OR 1.7 and 1.84 respectively). However, confidence interval values do not allow for an uncritical acceptance of those results. Similar outcomes were observed in relation to falls in the last 5 years (OR 1.03 and 3.92 respectively) (Table 6).

\section{Discussion}

Basic involutional changes associated with aging, which occur more rapidly in postmenopausal women, result in a decrease of parameters that assess muscle quality, i.e. muscle mass and function including strength and physical performance [2]. These parameters are used in clinical practice to diagnose sarcopenia, which often results in falls following fractures $[9,10]$.

Our own research shows more than a twofold increase in the risk of fall within a year in a group of 
women with a reduced muscle mass. Similar results, reported by Waters et al., showed a relation between balance loss and decreased ASMM index (HR 1.92; 95\% Cl 1.05-3.52) in women, which is most likely associated with an increased risk of falls. Additionally, subjects who have had sustained a fall at the start of a 4-year follow-up period had greater balance deficits when compared to those without a reported fall $(p=0.008)$ [13]. In the study by Scott et al. while analyzing mid-calf muscle density at the proximal tibia in a group of 2214 patients, the authors found a correlation between the reduction of density and increased risk of multiple falls in the following year (OR 1.62; 95\% Cl 1.16-2.23) [14]. In contrast to the above study by Van Ancum et al. reported a dependence between a reduced muscle mass, assessed with the skeletal muscle index (SMI) and falls in men alone. At the same time a decline of SMI index was not associated with a significantly increased risk of falls in the next 3 months following the initial muscle mass measurement [15]. Lack of an increased risk of falls in a group of 496 patients with a reduced muscle mass has also been reported when EWGSOP1 (HR 0.98; 95\% Cl 0.68-1.41) and Foundation for the National Institutes of Health Sarcopenia Project (FNIH) (HR 0.94; 95\% Cl 0.63-1.39) criteria were used [16].

Although the risk of falls was not found to be associated with a reduced muscle strength in our study, a prospective study with 496 subjects proved a relation between a decreased handgrip and recurring falls. The authors of the study observed a 1.5-fold (EWGSOP1 criteria, HR 1.51; 95\% Cl 1.01-2.25) and 2-fold (FNIH criteria, HR 2.05; $95 \% \mathrm{Cl} 1.26-3.33)$ increase in the risk of falls in those with a reduced handgrip [16]. Contrary to the above results, in a study on 378 subjects, the authors found that a decrease of handgrip $(p=0.02) \mathrm{did}$ not significantly increase the risk of falls in men (OR 0.93; 95\% Cl 0.88-0.99) in a 3-month follow-up. No such significant association was found between handgrip and falls in women [15]. A long lasting perspective on a risk of falls has been presented in a study by Balogun et al. where similar results were reported in relation to muscle strength. In a group of 1041 subjects there was no correlation between the lower values of handgrip and risk of falls in the last 10 years $(\beta 0.50 ; 95 \% \mathrm{Cl} 0.27$ 0.73). Handgrip was however found to be a predicator of subsequent fractures (RR 1.55; 95\% Cl 1.09-2.20) [17].

There is a limited number of studies that analyze the relation between physical performance and falls. In our study, as well as in the prospective study by Schaap et al., no significant association was observed between the increase of risk of falls and reduced physical performance assessed with gait speed tests using EWGSOP1 and FNIH criteria (HR 1.36, 95\% Cl 0.91-2.04; HR 1.46, $95 \% \mathrm{Cl} 0.99-2.16$ respectively) [16]. Contrary to that, a study by Scott et al. showed a significant correlation between the gait speed (OR 0.96; 95\% Cl 0.93-0.99), length of step $(0.87 ; 95 \% \mathrm{Cl} 0.77-0.98)$ and reduction of risk of falling in 3 years [18]

Sarcopenia as a progressive disorder of skeletal muscles has been directly linked, in multiple studies, with an increased probability of falls [19, 20], fractures, disability and mortality [9, 10, 19-25]. Therefore, early identification of patients with suspected sarcopenia is crucial to reduce the risk of the above-mentioned complications. Gadelha et al. stated that the frequency of falls significantly increases as the sarcopenia advances, reaching the highest spread of falls in those with severe sarcopenia [21]. Recent meta-analyses from 2019 and 2020 report an indisputable increase in the risk of falls in people over 65 years of age with sarcopenia $[19,20]$. The first meta-analysis, which included 10073 study subjects, presented a 1.5 -fold increase in the risk of falling in those with sarcopenia, when compared with the control group (OR 1.52; 95\% Cl 1.32-1.77) [19]. Similar results were observed in a 2020 meta-analysis, with a total of 52838 patients, where the prevalence of sarcopenia was associated with a higher risk of falls for women (OR 1.49; $95 \% \mathrm{Cl} 1.19-1.87)$ and men (OR 1.82; 95\% Cl 1.20-2.75) [20]. Both of the above-mentioned papers based their conclusions on research that had used different diagnostic criteria for sarcopenia. Bischoff-Ferrari et al. published a more detailed assessment by differentiating the risk of falls using various diagnostic criteria for sarcopenia. The authors stated that the largest increase may be observed when the criteria regarding decrease in muscle mass $\left(M<7.26 \mathrm{~kg} / \mathrm{m}^{2}\right.$; $\mathrm{W}<5.45 \mathrm{~kg} / \mathrm{m}^{2}$ ) by Baumgartner (OR 1.54; $95 \% \mathrm{Cl}$ 1.09-1.18) are used along with EWGSOP 2010 criteria (OR 1.82; $95 \% \mathrm{Cl} 1.24-2.69$ ). Cut-off points for muscle mass for both of the above-mentioned criteria are almost identical. Using these criteria, a higher risk is observed in women with sarcopenia (RR 1.97 vs. 2.81 respectively) than men (RR 1.10 vs. 1.31 respectively). When other criteria were implemented there was no correlation between sarcopenia and the increased risk of falls [26]. Two studies by Scott et al. from 2017 and 2019 confirmed an increased risk of falls (RR 1.58, 95\% Cl 1.14-2.17; RR 2.15, 95\% Cl 1.58-2.94 respectively) in patients with sarcopenia diagnosed using EWGSOP1 criteria, when compared with the control group (note: only male subjects were studied) [22, 23]. A similar relation was observed by Tanimoto et al. with a 4-fold increase of risk of falls in men (OR 4.42; $95 \% \mathrm{Cl} 2.08$ 9.39) and 2-fold in women (OR 2.34; 95\% Cl 1.39-3.94) diagnosed with sarcopenia using EWGSOP1 [24]. Sarcopenia diagnosed with these criteria also proved to affect risk of falling in a group of 260 patients (HR 3.25; $95 \% \mathrm{Cl} 1.54-6.88)$ after the study model had been adjusted by age and sex [25].

Contrary to the above results, our own study, along with a few others using EWGSOP1, did not prove an increased risk of falls in women with sarcopenia 
$[3,16,27]$. When FNIH criteria were applied in a study of 498 patients the authors found an increased risk of falls in subjects with sarcopenia (HR 2.38; 95\% Cl 1.37-4.14). FNIH cut-off points are considered more rigorous (ASMM $<5.12 \mathrm{~kg} / \mathrm{m}^{2}$, handgrip $<16 \mathrm{~kg}$, gait speed $<0.8 \mathrm{~m} / \mathrm{s}$ for women and ASMM $<7.89 \mathrm{~kg} / \mathrm{m}^{2}$, handgrip $<26 \mathrm{~kg}$, gait speed $<0.8 \mathrm{~m} / \mathrm{s}$ for men) than EWGSOP1 criteria [16]. Similar results with FNIH criteria were reported by Scott et al. with a 1.6-fold increase in the risk of falls for 2 years in men with sarcopenia [22]. Also when using the International Working Group on Sarcopenia (IWGS) criteria (men: ASMM $<7.23 \mathrm{~kg} / \mathrm{m}^{2}$, gait speed $<1.0 \mathrm{~m} / \mathrm{s}$; women ASMM $<5.57 \mathrm{~kg} / \mathrm{m}^{2}$, gait speed $<1.0 \mathrm{~m} / \mathrm{s}$ ) studies showed a 2.4 -fold increase of the risk of falls within a year (OR 2.41; 95\% Cl 1.03-5.56) in subjects with sarcopenia, contrary to the EWGSOP1 criteria (OR 1.62; 95\% Cl 0.41-6.31) [3]. It was estimated that approximately $10 \%$ of falls end up with a patient's hospitalization [11]; however, a study by Sim et al. with 903 women did not confirm increased hospitalization rates within 5 years in subjects with sarcopenia diagnosed either with FNIH (HR $0.93 ; 95 \% \mathrm{Cl} 0.51-1.70)$ or EWGSOP1 (HR 1.18; $95 \% \mathrm{Cl}$ $0.80-1.75$ ) [28]. To the best of our knowledge currently there is no study assessing the relation between risk of falls and sarcopenia using updated EWGSOP2 guidelines except the following - in which no increased risk of falls was reported within a year or 5 years. Although risk factors such as pain, single status, lack of social security and accompanying chronic diseases were found to be statistically significant in relation to the risk of falls [29], the authors did not include them in this paper.

Differences in the risk of falls among patients with sarcopenia in various studies may be explained by the use of different diagnostic criteria which had influenced the prevalence of sarcopenia. A review of the literature and our own study emphasize crucial differences in the epidemiology of sarcopenia depending on the diagnostic criteria that were used by the authors $[26,27,30-32]$. A meta-analysis that included 445 patients presents a prevalence of sarcopenia within the range of $2.5-27.2 \%$ in women and $3.1-20.4 \%$ in men depending on the criteria that were used [26]. A study by Kim et al. reports sarcopenia in $2.3-11.7 \%$ in women and $7.1-28 \%$ in men respectively using similar methodology [29]. Lowest sarcopenia prevalence was observed when FNIH criteria were applied (2.3\%) following IWGS (11.8\%) and EWGSOP1 guideline (13.3\%) [30]. Sarcopenia was more likely to be diagnosed when the definition was based on muscle mass alone. The above-mentioned criteria have evolved with time by broadening the definition with parameters such as muscle strength and physical performance. Low muscle mass as a sole risk factor does not have to affect physical performance and the onset of clinical symptoms [26, 29]. In our own study it was established that sarcopenia is 4 times more likely defined with EWGSOP1 criteria in compar- ison to the updated guideline from 2018 ( $p<0.001$ ). Even greater differences in the prevalence of sarcopenia using two sets of EWGSOP guidelines were reported by Petermann-Rocha et al. (8.14\% vs. $0.36 \%$ ) [31]. Contrary to that study, no significant differences (using both EWGSOP criteria) were reported by Rodriguez-Rejón et al. in a group of institutionalized patients [32]. Bianchi et al. reported that there were no significant differences in the prevalence of sarcopenia when EWGSOP2 (22.8\%) and FNIH (23.9\%) criteria were used [33]. Large discrepancies in the epidemiology of sarcopenia depending on the guidelines used indicate the need for further prospective studies on larger populations.

Despite the promising results of this study there are some limitations that should be mentioned, i.e. a small study sample $(n=122)$ and exclusion of 7 patients due to an incomplete questionnaire. Our study failed to assess the detailed relationship between coexisting comorbidities and drugs, which may have affected some of the results from the muscle quality assessment (i.e. handgrip, gait speed). Despite these limitations this is a preliminary study that assesses the relation between the risk of falls, the reduction of parameters evaluating the quality of skeletal muscles and sarcopenia. The study will continue in order to assess the problem in a larger study group.

\section{Conclusions}

1. The decrease of muscle mass is significantly correlated with the risk of falls in the last year in postmenopausal women, but due to the small study sample and heterogeneity of the available papers it is advised for the topic to be analyzed more thoroughly.

2. Risk of falls was not significantly increased for the falls in the last 5 years in those with decreased skeletal muscle quality parameters. This result has a lower empirical value as patients often fail to report a fall, most likely due to a problem with remembering an event from the distant past, especially if it was not associated with a trauma.

3. It was found that the impact of sarcopenia on the risk of falls depends on diagnostic criteria. An increased risk of falls was not proven in women with sarcopenia (using both EWGSOP1 and EWGSOP2). In order to clearly identify the relation between sarcopenia and increased risk of falls there is a need for studies with larger group samples, including meta-analysis using unified diagnostic criteria.

4. Prevalence of sarcopenia significantly differs depending on the type of diagnostic criteria that are used. Sarcopenia is more likely to be diagnosed with EWGSOP1 criteria, where muscle mass is a basic determining parameter (along with handgrip and/or physical performance), in comparison to the updated criteria EWGSOP2 from 2018 ( $p<0.001)$. 


\section{Disclosure}

The authors report no conflict of interest.

\section{References}

1. Dennison EM, Sayer AA, Cooper C. Epidemiology of sarcopenia and in sight into possible therapeutic targets. Nat Rev Rheumatol 2017; 13 340-347.

2. Auyeung TW, Lee SW, Leung J, et al. Age-associated decline of muscle, grip strength and gait speed: a 4-year longitudinal study of $3018 \mathrm{com}$ munity-dwelling older Chinese. Geriatr Gerontol Int 2014; 14: 76-84.

3. Clynes MA, Edwards MH, Buehring B, et al. Definition of sarcopenia: association with previous falls and fractures in a population sample. Calcif Tissue Int 2015; 97: 445-452.

4. Dodds RM, Syddall HE, Cooper R, et al. Grip strenght across the life course: normative data from twelve British studies. PLoS One 2014; 9 e113637.

5. Payette H, Gueyer NR, Gaudreau P, et al. Trajectories of physical function decline and psychological functioning: the Quebeck Longitudinal Study on Nutritoin and Successful Aging (NuAge). J Gerontol B Psychol Sci Soc Sci 2011; 66: 82-90.

6. Agostini D, Zeppa SD, Lucertini F, et al. Muscle and bone health in postmenopausal women: role of protein and vitamin D supplementation combined with exercise training. Nutrients 2018; 10: 1103-1124.

7. Shafiee G, Keshtkar A, Soltani A, et al. Prevalence of sarcopenia in the world: a systematic review and meta-analysis of general population studies. J Diabetes Metab Disord 2017; 16: 21.

8. Cruz-Jentoft AJ, Landi F, Schneider SM, et al. Prevalence of and intervention for sarcopenia in ageing adults: a systematic review; Report of the International Sarcopenia Initiative (EWGSOP and IWGS). Age Ageing 2014; 43: 748-759.

9. Cruz-Jentoft AJ, Baeyens JP, Bauer JM, et al. Sarcopenia: European consensus on definition and diagnosis. Age Ageing 2010; 39: 412-423.

10. Cruz-Jentoft AJ, Bahat G, Bauer J, et al. Sarcopenia: revised European consensus on definition and diagnosis. Age Ageing 2019; 48: 16-31.

11. Krysińska M, Domosławskie-Żylińska K, Fronk. Urazy wśród osób powyżej 60-go roku życia - Raport. Narodowy Instytut Zdrowia Publicznego - Państwowy Zakład Higieny, Warszawa 2017.

12. Wojtyniak B, Goryński P. Sytuacja zdrowotna ludności polski i je uwarunkowania. Narodowy Instytut Zdrowia Publicznego - Państwowy Zakład Higieny, Warszawa 2018.

13. Waters DL, Qualls CR, Cesari M, et al. Relationship of incident falls with balance deficits and body composition in male and female communitydwelling elders. J Nutr Health Aging 2019; 23: 9-13.

14. Scott D, Johansson J, McMillan LB, et al. Mid-calf skeletal muscle density and its association with physical activity, bone health and incident 12-moth falls in older adults: The Healthy Ageing Initiative. Bone 2019; 120: 446-451.

15. Van Ancum J, Pijnappels M, Jonkman NH, et al. Muscle mass and muscle strength are associated with-pre and post hospitalization falls in older male inpatients: a longitudinal cohort study. BMC Geriatr 2018 18: 116-123.

16. Schaap LA, van Schoor NM, Lips P, et al. Associations of sarcopenia definitions, and their components, with the incidence of recurrent falling and fractures: The Longitudinal Aging Study Amsterdam. J Gerontol A Biol Sci Med Sci 2018; 73: 1199-1204.

17. Balogun S, Winzenberg T, Wills K, et al. Prospective associations of low muscle mass and function with 10-year falls risk, incident fracture and mortality in community-dwelling older adults. J Nutr Health Aging 2017; 21: 843-848.

18. Scott D, McLaughlin P, Nichols GC, et al. Changes in gait performance over several years are associated with recurrent falls status in community-dwelling older women at high risk of fracture. Age Ageing 2015; 44: 287-293.

19. Zhang X, Huang P, Dou Q, et al. Falls among older adults with sarcopenia dwelling in nursing home or community: a meta-analysis. Clin Nutr 2020; 39: 33-39.
20. Yeung S, Reijnierse E, Pham V, et al. Sarcopenia and its association with falls and fractures in older adults: a systematic review and meta-analysis. J Cachexia Sarcopenia Muscle 2019; 10: 485-500.

21. Gadelha AB, Vainshelboim B, Ferreira AP, et al. Stages of sarcopenia and the incidence of falls in older women: a prospective study. Arch Gerontol Geriatr 2018; 79: 151-157.

22. Scott D, Seibel M, Cummings R, et al. Sarcopenic obesity and its temporal associations with changes in bone mineral density, incident falls, and fractures in older man: The Concord Health and Ageing in Men Project. J Bone Miner Res 2017; 32: 575-583.

23. Scott D, Seibel M, Cummings $R$, et al. Does combined osteopenia/ osteoporosis and sarcopenia confer greater risk of falls and fracture than either condition alone in older man? The Concord Health Study and Ageing in Men Project. J Gerontol A Biol Sci Med Sci 2019; 74: 827-834.

24. Tanimoto $Y$, Wantanabe $M$, Sun $W$, et al. Sarcopenia and falls in community-dwelling elderly subjects in Japan: defining sarcopenia according to criteria of the European Working Group of Sarcopenia in Older People. Arch Gerontol Geriatr 2014; 59: 295-299.

25. Landi F, Liperoti R, Russo A, et al. Sarcopenia as a risk factor for falls in elderly individuals: results from the ilSIRENTE study. Clin Nutr 2012; 31: 652-658.

26. Bischoff-Ferrari HA, Orav JE, Kanis JA, et al. Comparative performance of current definitions of sarcopenia against the prospective incidence of falls among community-dwelling seniors age 65 and older. Osteoporos Int 2015; 26: 2793-2802.

27. Kim H, Hirano H, Edahiro A, et al. Sarcopenia: prevalence and associated factors based on different suggested definitions in community-dwelling older adults. Geriatr Gerontol Int 2016; 16: 110-122.

28. Sim M, Prince RL, Scott D, et al. Utility of four sarcopenia criteria for prediction of falls-related hospitalization in older Australian women. Osteoporos Int 2019; 30: 167-176.

29. Sayilam A, Kulakac N, Saltan A. Determining the relationship between postoperative pain and the risk of falls in older adults. Pieleg Chir Angiol 2019; 4: 136-141.

30. Dam TT, Peters KW, Fargala M, et al. An evidence-based comparison of operational criteria for the presence of sarcopenia. J Gerontol A Biol Sci Med Sci 2014; 69: 584-590.

31. Petermann-Rocha F, Chen M, Gary SR, et al. New versus old guidelines for sarcopenia classification: What is the impact on prevalence and health outcomes? Age Ageing 2020; 49: 300-304.

32. Rodríguez-Rejón Al, Ruiz-López MD, Artacho Martín-Lagos R. Diagnosis and prevalence of sarcopenia in long-term care homes: EWGSOP2 versus EWGSOP1. Nutr Hosp 2019; 36: 1074-1080.

33. Bianchi L, Maietti E, Bellelli G, et al. Comparing EWGSOP2 and FNIH sarcopenia definitions: agreement and three-year survival prognostic value in older hospitalized adults. The Glisten Study. J Gerontol A Biol Sci Med Sci 2020; 75: 1331-133. 